\title{
All Substances (drugs) are Poisons; there is None which is not a Poison. The Right Dose Differentiates a Poison and A Remedy
}

Dear Readers,

Drug-induced hepatotoxicity is a potential adverse effect, contributing to the health burden, with several mechanisms involved in causing liver injury. The liver injury caused by the drug may vary with the extent of the damage, ranging from mild fatty liver to necrosis. Though uncommon and rare, it is contributing to morbidity and mortality in the general population and remains a potential complication for most of the prescribed drugs.

A prospective study conducted in the US have shown that $13 \%$ of the total cases were diagnosed as idiosyncratic hepatotoxicity; while $39 \%$ with acetaminophen (paracetamol) induced hepatotoxicity, however, it was interesting to know with the recent prevalence rates in south-east Asian registries, which revealed that $70 \%$ of the drug-induced liver injury cases occurred due to Herbal And Dietary Supplements. In India, antituberculosis drugs are contributing more to drug-induced liver injury.

Herbal preparations and dietary supplements may cause a large range of hepatic damage, resulting in liver failure. Epidemiological studies showed that $10-16 \%$ of the prevalence of herbal remedies associated with liver injury in Europe, the USA and other western countries. Hepatotoxicity associated with herbal preparations is difficult to demonstrate. There are difficulties in identifying toxicity; numerous factors play an important role. Frequent self-medication, assuming herbal remedies are not causing toxic effects and hence not disclosing the use of these herbal and dietary supplements to Physician. Another difficulty is that the actual composition of these herbal products remains unclear. Hence, clinical Pharmacists with consultation with Physicians may consider herbal and dietary products as potential contributing agents linked to hepatic injury.

It is important that clinical Pharmacists along with Physicians must be vigilant in recognizing drug-induced liver damage so that early detection can reduce the burden of hepatotoxicity if the drugs are discontinued. The manifestations of toxicity include elevation of hepatic enzymes and liver failure. Hence, it is necessary to have knowledge of the regularly prescribed drugs, herbal preparations and dietary supplements.
DOI: 10.5530/ijopp.14.4.48

Address for

correspondence:

Dr. Bhagya Venkanna Rao, Editor-in-chief, ijopp. Email Id: ijopp@emanuscript. in

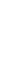

